\title{
Dreidimensionale Form- und Größencharaketrisierung von Erosionspartikeln mittels Röntgen-Computertomographie
}

\author{
Christian Rothleitner, Ulrich Neuschaefer-Rube, Jens Illemann \\ Physikalisch-Technische Bundesanstalt, Arbeitsgruppe 5.34 Multisensor-Koordinatenmesstechnik, \\ Bundesallee 100, 38116 Braunschweig, Deutschland
}

\begin{abstract}
Zusammenfassung
Es wird vorgestellt, wie mittels industrieller Röntgen-Computertomographie (CT) die Größe und die Form von Erosionspartikeln (z. B. Aluminiumoxid) mit Abmessungen größer als $20 \mu \mathrm{m}$ effizient bestimmt werden kann. Mehrere hundert bis tausend Partikel können gleichzeitig untersucht werden. Mit einer Standardsoftware zur Analyse von Volumendaten lassen sich die Sphärizität der Partikel nach Wadell in 3D charakterisieren. Die Größe der Partikel kann als volumenäquivalenter Durchmesser bestimmt werden, was einen direkten Vergleich mit gebräuchlichen Messmethoden, wie der Laserbeugung, zulässt.
\end{abstract}

Keywords: Röntgen-Computertomographie (CT), abrasive Mikropartikel, Form und Größe von Partikeln, 3-dimensionelle Messtechnik, Charakterisierung von Erosionspartikeln

\section{Einleitung}

Abrasive Mikropartikel treten in verschiedenen Bereichen der Wissenschaft und Technik auf. Micro Abrasive Blasting (MAB) [1] ist eine Methode, mit der mikromechanische Bauteile gefertigt werden können. Die Wirkung von abrasiven Partikeln kann also in der Technik gezielt genutzt werden. In den meisten Fällen jedoch ist der Effekt abrasiver Partikel negativ. Wenn Sandpartikel vom heißen Luftstrom einer Turbine mitgerissen werden, so können sie durch den Aufprall auf den Turbinenschaufeln Erosion versursachen. Dieser Abtrag von der Turbinenschaufel hat immense Kosten, aber auch sicherheitstechnische Konsequenzen für die Energie- und Luftfahrttechnik zur Folge. Bis dato ist der Mechanismus, welcher hinter der Erosion steckt, noch nicht im Detail verstanden. Vor allem für hohe Temperaturen (bis $900{ }^{\circ} \mathrm{C}$ ) und hohe Geschwindigkeiten (bis $300 \mathrm{~m} \mathrm{~s}^{-1}$ ) stehen bislang noch keine TestEquipments zur Verfügung. Die Entwicklung eines solchen Equipments hat sich das im „European Metrology Research Programme (EMRP)" geförderte Projekt "Metrosion" (Metrology to enable high temperature erosion testing) [2] zum Ziel gesetzt. Hier sollen Modelle entwickelt werden, die die Erosionsraten für hohe Temperaturen und hohe Geschwindigkeiten abschätzen. Dazu sollen experimentell ermittelte Daten verwendet werden. Ziel ist es, schließlich Informationen zu erhalten, die es ermöglichen, gezielt neue Materialien zu entwickeln, um Turbinenschaufeln vor Erosion zu schützen.

Neben Geschwindigkeiten und Temperaturen der Partikel sind Informationen über deren Größenverteilung und Formparameter wichtige Eingangsgrößen für Simulationen, denn einerseits hängt die Energie der Partikel von deren Größe ab und andererseits ist auch die "Kantigkeit", also die Form, ein wichtiger Erosionsparameter. Gebräuchliche Messmethoden, wie z. B. die Laserbeugung (Low Angle Laser Light Scattering, LALLS) basieren auf der Annahme eines kugelförmigen Partikels. Dies hat zur Folge, dass erstens die Form nicht bestimmt werden kann und zweitens das Volumen nicht genau gemessen werden kann. Mit dem Automated Dynamic Flow Particle Imaging hingegen lässt sich die Form relativ gut bestimmen, jedoch nur in zwei Dimensionen. Die Volumenmessung ist hier nur bedingt möglich und mit großen Messunsicherheiten behaftet.

Bis heute gibt es keine Messmethode, welche eine genaue Bestimmung von Form und Größe solcher Partikel in drei Dimensionen zulässt. An der PTB wurde nun ein Verfahren entwickelt, um Erosionspartikel in großer Anzahl auf diese Parameter hin zu analysieren. Dazu wurden Partikel mit einem industriellen Röntgen-Computertomographen (CT) gemessen und anschließend die relevanten Parameter mit einer weit verbreiteten Software bestimmt. Es wurden 
Partikel verschiedener Materialien untersucht, darunter Aluminiumoxid, Siliziumoxid, Chromoxid, aber auch Vulkanasche mit Partikeldurchmesser von etwa $20 \mu \mathrm{m}$ bis etwa $150 \mu \mathrm{m}$. Im Folgenden werden die Methode und Untersuchungsergebnisse anhand von Aluminiumoxid-Partikeln (Bezeichnung: Alumina Mesh 320) dargestellt. Die Partikel besitzen einen mittleren Durchmesser von ca. $40 \mu \mathrm{m}$.

\section{Grundlegende Betrachtung zu Größe und Form von Partikeln}

Bei der Angabe der Größe von Partikeln gibt es viele Möglichkeiten, die sich nach Messmethode oder Anwendungsgebiet richten [3]. Ein mögliches direktes Maß ist der FeretDurchmesser, der durch den Abstand zweier paralleler Tangenten definiert ist und mittels eines "Messschiebers" ermittelt werden könnte. Der größte so ermittelte Durchmesser $x_{\text {Fmax }}$ entspräche der Länge des Partikels (siehe [3]). Indirekte Größenangaben beziehen sich hingegen auf andere Eigenschaften des Partikels. Diese Eigenschaft wird dann mit einer Kugel ins Verhältnis gesetzt und schließlich der Kugeldurchmesser als Größe des Partikels definiert. Ist z. B. das Volumen des Partikels bekannt, so kann der Durchmesser einer Kugel angegeben werden, welche dasselbe Volumen enthält. Dies definiert den sogenannten volumenäquivalenten Durchmesser (siehe [3])

$$
x_{V}=\sqrt[3]{6 V / \pi} .
$$

Da diese Größendefinition das Volumen und falls die Dichte bekannt ist - auch die Masse repräsentiert, wurde sie für das Projekt ausgewählt. Die Masse ist für die Berechnung der kinetischen Partikelenergie notwendig.

Auch die möglichen Definitionen der Form eines Teilchens sind zahlreich. Da Ausgangspunkt vieler Simulationsmodelle (meist auf der Finiten-Elemente-Methode basierend) kugelförmige Partikel sind, bietet es sich an, als Korrektur solcher vereinfachten Modelle die Abweichung der Partikelform von einer Kugel anzugeben. Genau das ist in der Definition der Sphärizität $\Psi$ nach Wadell (siehe hierzu [3]) gegeben durch

$$
\Psi=\pi \frac{x_{V}^{2}}{S} .
$$

$S$ beschreibt hierbei die Oberfläche des Partikels. Die Sphärizität kann Werte zwischen null und eins annehmen, wobei eins einer Kugel entspricht. Wenngleich noch weitere Parameter für das Projekt relevant waren, sollen im Folgenden nur die beiden in (1) und (2) genannten Parameter betrachtet werden.

\section{Methode zur Bestimmung von Partikelform und -größe}

Die Partikel wurden in Form von Pulver bereitgestellt. Eine direkte Untersuchung mit CT ist in diesem Zustand jedoch nicht möglich. Denn die einzelnen Partikel lassen sich im rekonstruierten Volumen einer CT-Messung nicht voneinander trennen, bzw. besteht die Gefahr, dass die Separation mithilfe von Softwarealgorithmen fehlerhaft verläuft und somit Formabweichungen entstehen, welche zu falschen Formparametern führen würden. Es wurden daher die Pulverproben derart präpariert, dass die Partikel räumlich voneinander getrennt waren. Dies lässt sich durch Mischen des Pulvers mit Polymethylmethacrylat (PMMA) im Volumenverhältnis von mindestens 1:10 und anschließendes Aushärten erreichen. PMMA wurde zum einen als einbettende Matrix ausgewählt, weil es optisch transparent ist und sich das Präparat somit visuell inspizieren lässt. Zum anderen ist im rekonstruierten Volumen der Kontrast zwischen PMMA und den zu untersuchenden Partikelmaterialien hoch, was eine gute Oberflächenfindung ermöglicht.

Die präparierte Probe wurde mit einem industriellen Computertomographen (Nikon MCT 225) gemessen und anschließend das Volumen rekonstruiert. Ergebnis sind Volumendaten, die in Form von Grauwerten die räumliche Verteilung der Röntgenabsorption im Messvolumen angeben. Das Messvolumen setzt sich dabei aus Voxeln (volumetrischen Pixeln) zusammen. Auf die Rekonstruktion folgt die Oberflächenfindung, die größere Grauwertänderungen, wie sie an Oberflächen auftreten, findet und analysiert. Ein besonders einfaches Verfahren ist die sogenannte ISO50-Oberflächenfindung. Hier wird angenommen, dass sich die Oberfläche an den Orten befindet, an denen der Grauwert den Mittelwert der Grauwerte von Messobjektmaterial und umgebendem Material (in der Regel Luft) überschreitet. In der weit verbreiteten Software Volume Graphics Studio Max 2.2 (VGSM) [4] ist ein "erweiterter Modus" enthalten, bei dem die ISO50-Oberfläche nur der Startwert für eine genauere Oberflächenfindung ist. Diese Oberflächenfindungen (ISO50, bzw. ISO50+erweiterter Modus) arbeiten sub-voxel-genau. Das Volumen und der Oberflächeninhalt des gemessenen Volumens können daher mit hoher Genauigkeit bestimmt werden. Das Problem ist allerdings, dass mit dieser Genauigkeit nur das Gesamtvolumen, bzw. die Gesamtoberfläche aller sich im Messvolumen befindlichen Partikel ausgegeben wird. Für das Projekt ist es jedoch notwendig, die Parameter für jedes einzelne Partikel zu ermitteln. Außerdem soll die Analyse der Volumendaten in einer möglichst effizienten Weise erfolgen. Ein 
Aufteilen des Gesamtvolumens in Einzelvolumina, sogenannte „Regions of interest (ROI)“, mit je nur einem Partikel, ist zwar machbar, jedoch sehr zeitaufwändig, da nicht automatisierbar. Ein effizienter Weg war, das Zusatzmodul Defektanalyse der Software VGSM zu verwenden. Hierbei werden die Partikel als Einschlüsse im Grundmaterial "Defekte" des PMMA analysiert, wie dies in der Materialwissenschaft häufig durchgeführt wird. Das Zusatzmodul separiert und indiziert die einzelnen Defekte automatisch und gibt dazu jeweils verschiedene Parameter an, darunter auch die im Projekt erforderlichen Grundgrößen Volumen und Oberflächeninhalt.

\section{Messunsicherheitsbetrachtung}

Derzeit gibt es keinen „Partikelstandard“, welcher als Referenz für diese Messungen herangezogen werden könnte. Da auch gebräuchliche Messmethoden, wie das LALLS, ebenfalls nur auf Modellen basieren, können diese nicht als Referenz herangezogen werden. Außerdem wäre bestenfalls ein statistischer Vergleich möglich, nicht aber ein 1-zu-1-Vergleich eines jeden gemessenen Partikels. Deshalb wurde eine eigene Strategie entwickelt, die in Abb. 1: schematisch dargestellt ist. Zuerst wurden synthetische Objekte (Regelgeometrien) mit wohldefiniertem Volumen und Oberfläche erzeugt. Mit diesen Objekten werden dann CT-Messungen simuliert. Hierfür wird das Programm aRTist [5] verwendet. Die rekonstruierten Volumendaten werden sodann in VGSM geladen und die Oberfläche im ISO50+erweiterten Modus gefunden. PMMA betrachtet und diese somit als

jeden Objektes ausgelesen. Da die Nominalwerte der Objekte bekannt sind, kann die Genauigkeit dieser Methode abgeschätzt werden. Die relativen Abweichungen vom Nominalwert betragen hier unter $1 \%$. Die Methode der sub-voxel-genauen Parameterbestimmung (im Folgenden einfach ISO50+erweiterter Modus genannt) kann nachfolgend als Referenz benutzt werden. Durch einen Vergleich der Parameter aus beiden Auswertemethoden lässt sich dann auch die Genauigkeit der Defektanalyse ermitteln. Es stellte sich hierbei heraus, dass mit dem Modul Defektanalyse die Oberflächen mit Fehlern von bis zu $50 \%$ zu groß ermittelt werden. Dies liegt daran, dass der Algorithmus bei Defektanalyse nur mit Voxel-Präzision arbeitet. Da aber die Defektanalyse deutlich effizienter ist, soll diese zur Auswertung aller gemessenen Partikel verwendet werden. Eine Korrektur lässt sich dann statistisch ermitteln. Dazu wird aus allen Partikeln des Messvolumens eine noch handhabbare Untermenge von ca. 100 Partikeln entnommen und in separate ROls aufgeteilt. Wie bei den Regelgeometrien werden die Parameter Volumen und Oberfläche aus der Defektanalyse und dem ISO50+erweiterten Modus wieder verglichen. Ist diese Untermenge repräsentativ, so können die Mittelwerte aus der Defektanalyse korrigiert werden. Somit lässt sich nun das gesamte Messvolumen mit Defektanalyse untersuchen und die Mittelwerte korrigieren.

Die vorangehende Betrachtung gibt nur die relativen Fehler der Defektanalyse im Vergleich zur sub-voxel-genauen Oberflächenfindung (ISO50+erweiterter Modus) an. Um eine Messunsicherheitsangabe nach GUM [6] anzugeben, wäre eine genaue Analyse des CT-Systems und des Messprozesses notwendig. Bei den geringen Dimensionen der Partikel ist jedoch der größte Unsicherheitsbeitrag durch die Oberflächenfindung selbst bestimmt. Auf Erfahrungswerten basierend schätzen wir die Unsicherheit der gefundenen OberAnschließend werden die Objekte sowohl mit dem Modul "Defektanalyse" untersucht, als auch einzelne Partikel in separaten Regions Of Interest (ROIs) gemessen und aus deren "Eigenschaften" das mit Sub-Voxel-Präzision berechnete Volumen und die Oberfläche eines flächenposition auf einen viertel Voxel, was mittleren Partikeldurchmesser von $40 \mu \mathrm{m}$, ergibt das eine relative Standardmessunsicherheit von $2,5 \%$ für den Durchmesser und für das Volumen $7,5 \%$. Diese relative Standardetwa 0,5 $\mu \mathrm{m}$ entspricht. Bezogen auf einen 
messunsicherheitsbetrachtung stellt jdoch nur eine vorläufige dar. Eine detailliertere Analyse ist geplant.

\section{Verbesserung der Oberflächenbestimmung im Modul Defektanalyse}

Neben Volumen und Oberfläche der Defekte gibt das Modul Defektanalyse noch viele weitere Parameter aus, wie z. B. die Projektionsflächen des Defekts auf die drei Grundflächen ( $P X, P Y$ und $P Z$ ) des Koordinatensystems. Dies stellt sich als sehr hilfreich heraus, um die Oberflächen der Partikel genauer anzugeben, als dies von der Defektanalyse im Standardverfahren getan wird. Ein Theorem von Cauchy besagt nämlich, dass sich die Oberfläche eines konvexen Objekts näherungsweise als

$$
S=4 \cdot \bar{A}
$$

berechnen lässt. Hierbei beschreibt $\bar{A}$ den Mittelwert der projizierten Flächen. Diese lässt sich näherungsweise mit

$$
\bar{A}=(P X+P Y+P Z) / 3
$$

berechnen. Eine Anwendung des Theorems auf die synthetischen Regelgeometrien ergab Abweichungen von weniger als $6 \%$ vom Nominalwert, also eine erhebliche Verbesserung im Vergleich zu den $50 \%$ des gebräuchlichen Verfahrens.

\section{Ergebnisse}

Eine Probe von Alumina Mesh 320-Partikeln wurde mit dem CT-System Nikon MCT 225 tomographiert und anschließend rekonstruiert. Das CT-System besitzt ein Reflektionstarget aus Wolfram und einen Detektor mit $2000 \times 2000$ Pixeln à $200 \mu \mathrm{m}$ Kantenlänge. Die gewählten Messparameter sind in Tab. 1: dargestellt.

Tab. 1: Einstellungen für die CT-Messung.

\begin{tabular}{|l|l|}
\hline $\begin{array}{l}\text { Geschätzte } \\
\text { Brennfleckgröße }\end{array}$ & $5 \mu \mathrm{m}$ \\
\hline Spannung/Strom & $85 \mathrm{kV} / 82 \mu \mathrm{A}$ \\
\hline $\begin{array}{l}\text { Anzahl der } \\
\text { Projektionen }\end{array}$ & 2360 \\
\hline $\begin{array}{l}\text { Filter Material / } \\
\text { Dicke }\end{array}$ & $\begin{array}{l}\text { Aluminium / 0,1 mm } \\
\text { (plus 0,6 mm } \\
\text { Detektorschutzhülle) }\end{array}$ \\
\hline Voxelgröße & $1,94 \mu \mathrm{m}$ \\
\hline Scanzeit & 2,6 Stunden \\
\hline
\end{tabular}

Abb. 2: zeigt sowohl rekonstruierte Partikel der Probe (Abb. 2a), als auch ein einzelnes Partikel (Abb. 2b). Die Form kann, trotz der geringen Größe, sehr gut aufgelöst werden. Das gesamte gemessene Volumen enthielt 1418 Partikel. Eine Untermenge von 119 Partikeln wurde in separate ROls aufgeteilt, um den Vergleich zwischen Defektanalyse und
ISO50+erweiterter Modus herzustellen. Das bedeutet, dass jedes dieser 119 Partikel mit beiden Methoden untersucht wurde und ein direkter 1-zu-1-Vergleich möglich war. Die Auswertung der Oberflächen mit der Defektanalyse erfolgte dabei mit dem verbesserten Verfahren, d. h. durch Anwendung des Cauchy Theorems. Die Ergebnisse des Vergleichs sind in Abb. 3: dargestellt. Die Defektanalyse unterschätzt dabei sowohl das Volumen $(-3,71 \%)$, als auch die Oberfläche (-4,02 \%).

a)

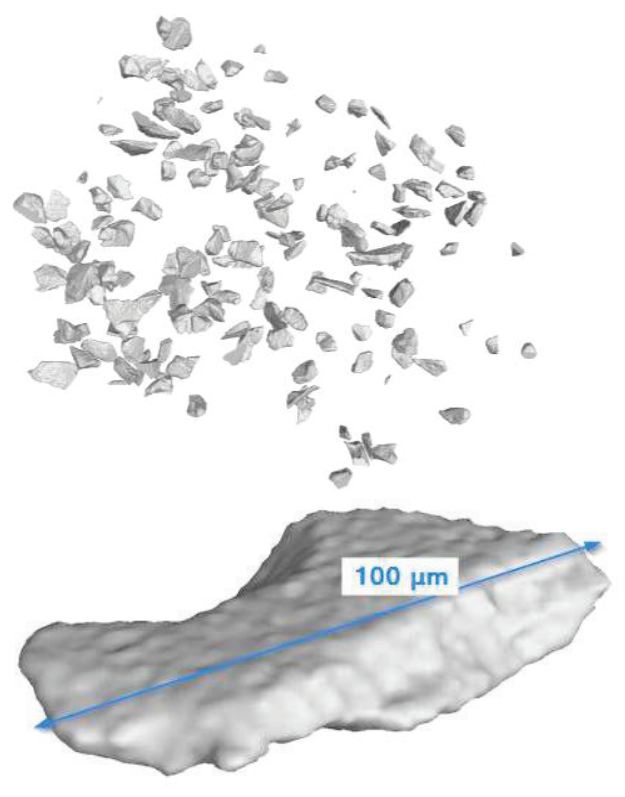

Abb. 2: a) Messergebnis der Probe mit Aluminiumoxid-Partikeln.

b) Einzelnes rekonstruiertes Aluminiumoxid-Partikel.

Für den volumenäquivalenten Durchmesser resultiert daraus eine relative Abweichung von $-1,26 \%$ und für die Sphärizität nach Wadell $+1,75 \%$ (siehe Abb. 4:). Durch Anwenden der Defektanalyse auf alle 1418 Partikel ergab sich ein mittlerer volumenäquivalenter Durchmesser von $x_{\mathrm{v}}=37,95 \mu \mathrm{m}$ (siehe auch Abb. 4: und Abb. 5:). Die Korrektur dieses Wertes aufgrund des vorhergehenden Vergleichs führt schließlich zum Wert $x_{V \text {, korrigiert }}=38,43 \mu \mathrm{m}$. Für die Sphärizität ergibt sich $\Psi=0,74$ bzw. $\Psi_{\text {korrigiert }}=$ 0,73 (siehe hierzu Abb. 4: und Abb. 5:).

\section{Vergleich mit LALLS}

Eine Methode zur Messung der Größenverteilung von Partikeln in dem für uns relevanten Größenbereich, die dem Stand der Technik entspricht, ist das Low Angle Laser Light Scattering (LALLS). Um zu ermitteln, inwieweit die Resultate einer solchen Messung mit unseren Ergebnissen übereinstimmen, haben wir aus derselben Pulverprobe Partikel mit diesem Verfahren untersuchen lassen. Dazu wurde ein Mastersizer 2000 von Malvern Instruments Ltd verwendet [7]. 


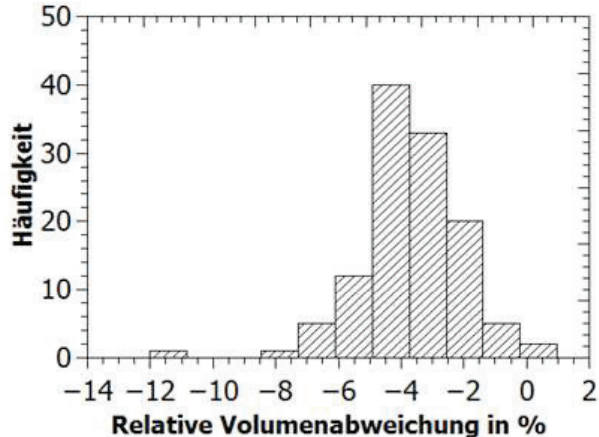

a)

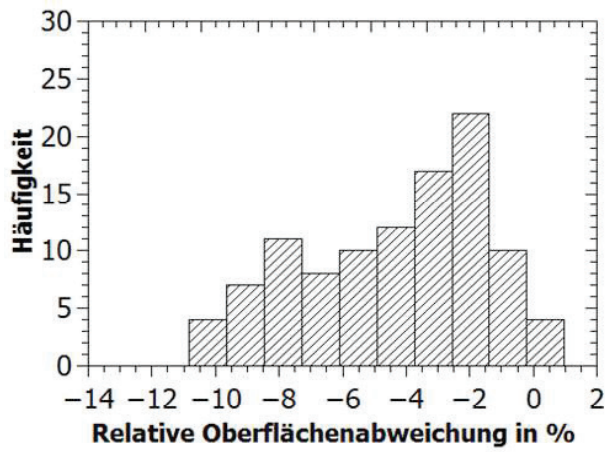

Abb. 3: Vergleich der Parameter Volumen (a) und Oberfläche (b) zwischen Defektanalyse und ISO50+erweiterter Modus zeigt, dass Defektanalyse beide Parameter unterschätzt.

Die Messung ergab einen Median für den volumeäquivalenten Durchmesser von $\mathrm{D}_{0.5}=49,0 \mu \mathrm{m}$. Verglichen $\mathrm{zu}$ dem von uns ermittelten Wert $x_{V \text {, korrigiert }}=38,4 \mu \mathrm{m}$ (der Mittelwert ist in unserer Messung etwa gleich dem Median), bedeutet dies eine relative $A b-$ weichung der LALLS-Messung um $+28 \%$ von unserem Ergebnis. Die kinetische Energie der Partikel würde mit LALLS somit um einen Faktor 2,1 höher berechnet werden. Unsere Messunsicherheitsabschätzung von 2,5\% im Durchmesser der Partikel ist zwar nur vorläufig, es kann jedoch angenommen werden, dass unsere Messmethode den wahren Wert der Partikelparameter besser trifft. Unsere Methode berücksichtigt die Oberflächenstruktur der Partikel wesentlich besser als dies beim LALLS getan wird. Bei LALLS wird ja von einer sphärischen Partikelform ausgegangen. Zudem ist die Lichtstreuung an unregelmäßigen Partikeloberflächen sehr komplex und deshalb schwer zu modellieren.

\section{Zusammenfassung und Ausblick}

Abrasive Partikel in der Größenordung von wenigen Mikrometern bis über hundert Mikrometer erzeugen in den meisten Fällen negative Effekte, wie den Abtrag von Material, z. B. von Turbinenschaufeln oder Gas-, bzw. Ölpipelines. Die Wirkung von abrasiven Partikeln kann jedoch in der Technik auch gezielt genutzt werden. Beispielsweise ist Micro Abrasive Blasting (MAB) [1] eine Methode, mit

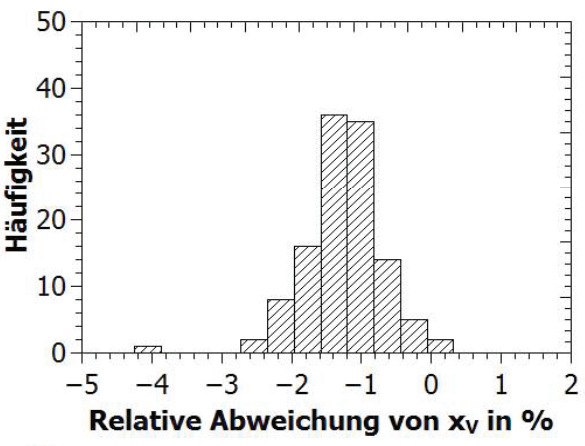

a)

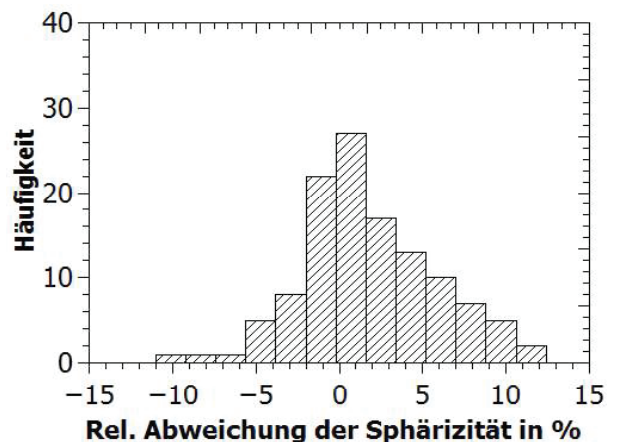

b)

Abb. 4: Vergleich der Auswertungen mithilfe von RIOs und durch Anwenden des Cauchy-Theorems auf die Ergebnisse des Moduls Defektanalyse: Der volumenäquivalente Durchmesser $x_{V}$ wird mit der Defektanalyse unterschätzt (a), während die Sphärizität nach Wadell überschätzt wird (b).

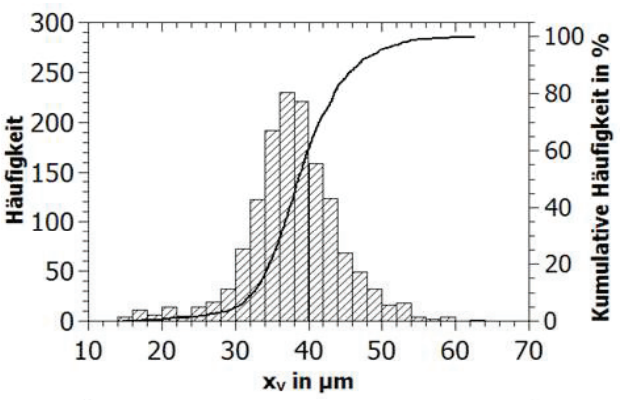

a)

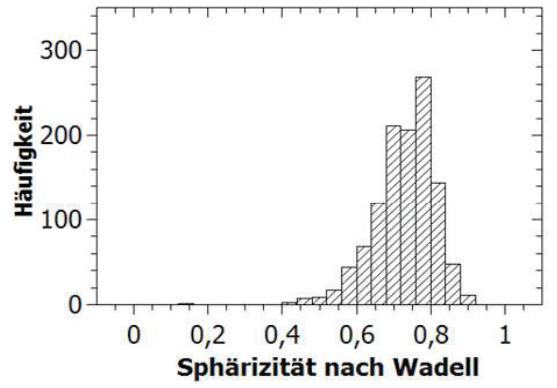

Abb. 5: Häufigkeitsverteilung aller 1418 Partikel, ermittelt mit Defektanalyse (Anwendung von Cauchys Theorem): a) Volumenäquivalenter Durchmesser. b) Sphärizität nach Wadell.

der mikromechanische Bauteile gefertigt werden können. Um die gewollten oder un- 
gewollten Effekte besser zu verstehen, ist es in jedem Fall wichtig, gewisse Partikelparameter so genau wie möglich zu kennen. Dies ermöglicht die Entwicklung verbesserter Modelle und schließlich neuer Materialien, welche gefährdete Bauteile vor ungewollter Erosion schützen.

In diesem Artikel wurde eine neue effiziente Methode vorgestellt, mit der sich submillimetergroße abrasive Partikel in großer Anzahl und in 3 Dimensionen auf Größe und Form untersuchen lassen. Dazu wurde eine Pulverprobe so präpariert, dass die einzelnen Partikel räumlich getrennt voneinander fest in eine PMMA-Matrix eingebettet sind. Die präparierte Probe wurde anschließend mit einem industriellen Computertomographen gemessen. Mithilfe einer weit verbreiteten Software konnten aus den rekonstruierten Daten Parameter wie Volumen und Oberfläche effizient ermittelt werden. Eine Ungenauigkeit bei der effizienten Oberflächenberechnung konnte durch Anwenden des Cauchy Theorems für konvexe Körper von $50 \%$ auf $6 \%$ reduziert werden. Diese effiziente Methode wurde dabei gegen eine hochgenaue, aber ineffiziente Methode verifiziert.

Eine vorläufige relative Standardmessunsicherheit konnte durch Abschätzen der Abweichungen bei der Oberflächenfindung der rekonstruierten Messdaten angegeben werden. Es folgt, dass die CT-Messung den volumenäquivalenten Durchmesser der Partikel auf etwa 2,5\% und das Volumen der Partikel auf etwa $7,5 \%$ genau angeben kann. Ein Vergleich der CT-Methode mit einer gängigen Methode, die auf der Laserbeugung basiert, zeigt, dass diese den Partikeldurchmesser für die untersuchten Partikel um $28 \%$ überschätzt. Eine Folge wäre, dass die kinetische Energie der Partikel um einen Faktor 2,1 zu hoch angenommen wird, was $\mathrm{zu}$ falschen Simulationsmodellen führt.

Die von uns vorgestellte Messmethode besitzt ein hohes Potential einen neuen Standard in der Partikelmesstechnik einzunehmen. Dazu sind jedoch weitere Untersuchungen notwendig und auch geplant.

\section{Zusammenfassung und Ausblick}

Die Autoren danken Dr. Federico Cernuschi, von RSE S.p.A. in Mailand/ Italien, für die Auswertungsergebnisse mit dem LALLS.

This work was funded through the European Metrology Research Programme (EMRP) Project IND61 Metrosion. The EMRP is jointly funded by the EMRP participating countries within EURAMET and the European Union.

\section{Literaturnachweis}

[1] E. Belloy, S. Thurre, E. Walckiers, A. Sayah and M. Gijs, "The introduction of powder blasting for sensor and microsystem applications," Sensors and Actuators A: Physical, vol. 84, no. 3, pp. 330-337, 2000.

[2] "Metrology to enable high temperature erosion testing (METROSION)," European Metrology Research Program (EMRP), 2015. [Online]. Available at: http://projects.npl.co.uk/metrosion/.

[3] ISO, "9276-6: 2008 (E) Descriptive and quantitative representation of particle shape and morphology", International Organization for Standardization, Geneva, 2008.

[4] Volume Graphics GmbH, "VG Studio Max 2.2," Software, November 2015. [Online]. Available at: http://www.volumegraphics.com/de/.

[5] Bundesanstalt für Materialforschung und prüfung (BAM), "aRTist - Analytical RT Inspection Simulation Tool," Software, December 2015. [Online]. Available at: http://www.artist.bam.de/.

[6] BIPM et al., "Evaluation of measurement data-Guide to the expression of uncertainty in measurement (GUM)," Geneva, 2008.

[7] Malvern Instruments Ltd, "Mastersizer 2000," November 2015. [Online]. Available at: http://www.malvern.com/en/support/productsupport/mastersizer-range/mastersizer2000/default.aspx. 